\title{
LA OBRA ENCICLOPÉDICA DE JUAN ANDRÉS Y LA ESCUELA UNIVERSALISTA ${ }^{1}$ \\ THE ENCYCLOPEDIC WORK OF JUAN ANDRÉS AND THE UNIVERSALIST SCHOOL
}

\author{
Pedro Aullón de Haro \\ Universidad de Alicante, España \\ padeh@ua.es \\ Davide Mombelli \\ Universidad de Alicante, España \\ davide.mombelli@ua.es
}

\begin{abstract}
Resumen:
El presente artículo constituye una introducción a la Escuela Universalista Española del siglo XVIII, tanto en sentido conceptual como de disposición de autores, esferas y círculos de relación, individualidades y subescuelas temáticas o disciplinares. Se especifican pues las características fundamentales de la Ilustración universalista y sus semejanzas y diferencias epistemológicas respecto de las demás Ilustraciones europeas.
\end{abstract}

Palabras clave: Escuela Universalista, genealogías, disciplinas, Ilustración Universalista

\begin{abstract}
:
This article constitutes the basic layout of the Escuela Universalista Española of $18^{\text {th }}$ century, both in the conceptual sense and the disposition of authors, spheres and circles of relationship, individualities and thematic or disciplinary subscales.The fundamental characteristics of the Universalist Enlightenment and its epistemological similarities and differences with respect to the other European Enlightenments are here specified.
\end{abstract}

Keywords: Escuela Universalista, genealogies, disciplines, Universalist Enlightenments

Recibido: 14 de marzo de 2018

Aprobado: 30 mayo de 2018

\section{I}

Juan Andrés (Planes, Alicante 1740 - Roma 1817), humanista y científico, talento múltiple y cabeza de escuela, celebérrimo en la Europa de su tiempo es el ideador o director del Primer Programa de la Escuela Universalista Española o

\footnotetext{
${ }^{1}$ La presente publicación es resultado del proyecto "La Escuela Universalista Española del siglo XVIII y la creación de la Comparatística moderna", Ministerio de Economía y Competitividad, Referencia FFI2015-65769-P (MINECO/FEDER).
} 
Hispánica: Prospectus Philosophiae Universae (Aullón, Escuela 45-62). Las obras principales de Juan Andrés, creador de la Historia universal y comparada de las Letras y las Ciencias, Origen progresos y estado actual de toda la literatura; Lorenzo Hervás, autor del Catálogo de las lenguas, que establece los fundamentos de Lingüística universal y comparada; Antonio Eximeno, Del origen y reglas de la Música, que sitúa este arte mediante el concepto de expresión como lenguaje universal, son emblema de la Escuela Universalista. En ellas confluye la creación de una Ilustración científica y humanística, cristiana y no política, hasta ahora escamoteada, la cual constituye la creación de la Comparatística moderna.

La vida de Juan Andrés, entre los momentos inicial y final, de nacimiento y muerte, describe tres épocas. En primer lugar la de formación española, que culmina en el desempeño de la cátedra de Retórica en la Universidad de Gandía ${ }^{2}$, y concluye con la expulsión de España de la orden ignaciana en 1767. Entre la Universidad de Gandía y la Biblioteca de Nápoles, vivió Andrés los más de veinte años de plenitud, trabajo y satisfacción humana que representa la época de Mantua. En tercer lugar, la difícil época de la Biblioteca de Nápoles, donde el ejercicio de sus funciones le condujo a importantes logros bibliotecológicos, pero donde padeció la insidia y sufrió la pérdida de la vista hasta el final de sus días.

Las tres épocas, desafortunadamente, estuvieron jalonadas por transiciones dolorosas, de destierro, huida y desafección, empezando por una expulsión inhumana y de larga espera en Córcega hasta por fin alcanzar el colegio de Ferrara con sus compañeros de orden; en segundo lugar la larga huida de Mantua, y la itinerancia a que ésta le condujo,consecuencia de la llegada de las tropas napoleónicas; y en tercer lugar el viaje último de un hombre ciego de Nápoles a Roma, finalmente calumniado pero habiendo dejado escrita la historia de la Biblioteca de Nápoles y en ella la crónica del largo expolio a que había sido sometida (Andrés, Estudios 159-235).

La época mantuana, la más feliz y productiva, viene definida en el ámbito privado por la presencia de la familia de los marqueses Bianchi, que le acoge en Palacio como preceptor; y en el ámbito público por la Accademia de Mantua, que le abre el camino de la vida intelectual italiana. Intelectualmente, Mantua sobre todo significa, tras la redacción del Primer Programa filosófico y científico, fruto de la breve estancia previa en el Colegio de Ferrara, la elaboración de la gran obra enciclopédica de

\footnotetext{
${ }^{2}$ Pionera universidad jesuita, actualmente desaparecida.
} 
Andrés: la Historia universal de las Letras y las Ciencias, respuesta a la Enciclopedia francesa, compuesta por un solo hombre y sustentada en una epistemología o taxonomía de las ciencias que sostiene la historia como fórmula y la comparatística como método. Con ésta accede Andrés a una celebridad internacional que le convierte en gran referencia intelectual europea durante el último cuarto del siglo XVIII y comienzos del XIX. En 1836-1839 se realiza en Nápoles la última y póstuma edición completa de la Historia de Andrés. La Escuela Universalista también lo es hispano-italiana pues en gran medida se trata de obras escritas originalmente en Italia y en lengua italiana.

La expulsión de los jesuitas significó, como es sabido, el progresivo establecimiento en Italia de un nutrido y muy relevante grupo intelectual de jesuitas españoles y en general hispánicos. Pero el hecho importante viene determinado por la creación en Italia y en lengua italiana de una corriente o escuela intelectual o científica de tendencia universalista y extraordinarias dimensiones que sólo recientemente ha sido posible reconstruir y ha puesto sobre la mesa la necesidad de reescribir la historia moderna del pensamiento. De algún modo, Andrés era consciente de la dimensión fáctica de esta corriente, según se puede comprobar desde las primeras páginas de sus Cartas familiares.

Esta Escuela Universalista, como veremos, consiste en una nutrida serie de autores, en su mayoría jesuitas expulsos, no sólo de España sino también de ultramar, América y Filipinas. Excepto algunos de ellos, en especial los naturalistas y botánicos (Celestino Mutis, Antonio José Cavanilles, Franco Dávila, los jesuitas Francisco Javier Clavijero y Juan Ignacio Molina...) apenas han sido estudiados. Es de subrayar la gran dimensión no sólo humanística sino también científica, físico-matemática, de la Escuela, la cual por otra parte presenta en último término una interesante y notabilísima derivación meteorológica y sismológica (Benito Viñes en La Habana y Federico Faura en Manila, que a su vez formaron escuela) (Aullón, Constitución 43-70).

Todo ello tiene una genealogía teórica en el marco del pensamiento humanístico del parangón que esquemáticamente cabría reducir a una tríada constructiva históricamente representada por Dionisio de Halicarnaso, Julio César Scalígero y Daniel Georg Morhof, la cual es conducida a plenitud por los universalistas españoles en Italia. Se trata, pues, como hemos dicho, de la construcción de la Comparatística moderna (Aullón, Metodologías 291-300). 
Andrés transita con normalidad, aun implícitamente, del concepto clásico humanístico a la epistemología ilustrada, pero en ese tránsito configura precisamente el sentido de las ciencias y de la historia que el enciclopedismo francés había destruido. Esta operación se efectúa gracias al criterio historiográfico y al método comparatista. Ciertamente, el desafío de Andrés a la Enciclopedia francesa es mucho más que de capacidad y fuerzas de "un solo hombre", es un desafío de gran alcance epistemológico cuyo alcance sólo actualmente es plenamente reconocible.

El pensamiento humanístico clásico, en cuyo seno nace la teoría comparatista, la alianza de saber, moral y dignidad humana asociada a esa metodología así como la necesaria amplitud generalista para la comprensión y mejora del mundo y la correspondiente formación de la ciencia, se encuentra a la base de toda posible teoría o visión universalista, o al menos la integradora y fundada en esos presupuestos aquí por nosotros preconizada. Es decir, la concepción universalista no es sino una expansión especial de la teoría del humanismo que accedería a su clímax a finales del siglo XVIII alcanzando su más característica y potente realización. Pero sucede además que esto presenta un relieve de primer orden capaz de entregar medios e infundir sentido a la época de la Globalización.

Se trata de la formación de una Ilustración mediante la instrumentación metodológica incrementada a sumo grado de la tradición comparatística. La creación de la Comparatística moderna se efectúa, como dijimos, a partir de la tradición grecolatina y renacentista ahora reconstituida con criterios de universalidad, por cuanto únicamente una adecuada interpretación de la idea de universalidad hace posible el acceso a una epistemología comparatista bien constituida, y si 'universalidad' es concepto, por así decir, pluricategorial a tenor de los planos susceptibles de consideración, el comparatismo conforma el único posible camino de acceso a la universalidad y a su centro metodológico. El acceso de Juan Andrés, o Lorenzo Hervás, a la universalidad presupuso la asunción de la particularidad, de las partes del todo. La analogía de la universalidad con el conocimiento empírico del planeta Tierra todo significa un momento, último en su plano, de la escala de ese conocimiento, evidentemente clave final de tal escala, la cual presupone totalidad del orbe y, por ende, de las ciencias y las letras de las disciplinas y saberes. Como ejecución práctica es el trayecto que va de Plinio a Juan Andrés. Esto se comprenderá que es singularmente decisivo para la interpretación constructiva de la era de la Globalización, ayuna de contenido y 
proyectada como mera inercia profundamente inconsciente de sí misma (Aullón, Metodologías 291-311).

Pero se trata, asimismo, históricamente, de la construcción de una Ilustración científica y humanística, historiográfica y no política cuya explanación vino a culminar una nueva perspectiva de cosas. Es lo que hemos convenido en denominar Escuela Universalista del siglo XVIII, española, hispánica o hispano-italiana. Esta nutrida Escuela, constituida decisoriamente mediante criterios científicos empiristas al tiempo que en la asimilación y reelaboración del humanismo clásico, constituye un gran complemento y una alternativa los cuales exigen para nuestro tiempo la reconstrucción de la Historia de las ideas y en general del pensamiento y la ciencia moderna. Es necesaria, pues, la reinterpretación de la Ilustración europea y la determinación de un aspecto de la misma fundado por sí en la emancipación cultural y científica como progreso al margen de la revolución política. A pesar de la mucha distancia, en esto, y en la asimilación directa de ética y disciplina, se asemejan la Escuela Universalista y el pensamiento estético de valor más general, de Friedrich Schiller.

La producción del jesuita ilustrado Juan Andrés define en su conjunto una extensa obra enciclopédica de la Ilustración, de materia humanística y científica, publicada originalmente entre los siglos XVIII y XIX, en Italia y España. Aparte de sus dos obras más extensas y difundidas, la referida Historia universal de las Letras y las Ciencias (Origen, progresos y estado actual de toda la literatura) y la también mencionada colección epistolar de su Viaje de Italia (Cartas familiares), Andrés es también autor de una veintena de escritos de diversa naturaleza, dimensión y transcendencia que arrancan del inicial Prospectus Philosophiae Universae, ya referido y que próximamente verá la luz en edición bilingüe.

Se trata de escritos tanto humanísticos como científicos y bio-bibliográficos. El resto de la producción de Andrés se limita a ciertos manuscritos no publicados en vida del autor. De estos manuscritos han sido editados recientemente dos importantes, los pertenecientes a la Accademia de Mantova; los restantes están siendo estudiados en la actualidad $^{3}$. A ello, por otro lado, se ha de sumar el epistolario privado, al menos en buena parte ya impreso (Andrés, Epistolario).

En general, la obra de Andrés configura, si bien se mira, un lugar de privilegio a fin de observar la constitución moderna del gran segmento de los géneros ensayísticos y

\footnotetext{
${ }^{3}$ Esta labor, asumida por el Instituto Juan Andrés, es actualmente desempeñada por Davide Mombelli.
} 
la extensa problemática que desde el siglo XVIII para la ciencia literaria esto constituye y aún no ha sido plenamente afrontado. A ello precisamente cabría referirse tomando el excepcional ejemplo de Anécdota Graeca et Latina. Por lo demás, es necesario entender y asumir el hecho de que la obra de Andrés fue víctima, en sumo grado, primero, de la falacia de un Romanticismo que quiso sobreponerse a la superioridad ilustrada en materia historiográfica (vid. Cassirer) ${ }^{4}$; segundo, fue víctima de la subsiguiente postergación de la cultura hispánica y cristiana por parte de las evoluciones sociopolíticas y parcialistas de la propia Ilustración; tercero, fue víctima de los ámbitos, sobre todo francés y norteamericano, de la Literatura Comparada, deseosos de borrar su pasado fundacional moderno, y ello hasta el punto de interrumpir el conocimiento y caer en el olvido de su propio pasado aún anterior, clásico, grecolatino y renacentista. Con posterioridad se ha querido asimilar disminuidamente su entidad, reducida a mera tradición erudita eclesiástica, por parte de los estudios histórico-sociológicos, pero ello es de notar que ejecutado desde la más cruda omisión o ignorancia de la historia del pensamiento y las ideas.

Andrés escribió en lenguas italiana, española y latina, por este orden cuantitativamente de mayor a menor. No escribió, nunca, en ninguna otra lengua. Fue traducido, sobre todo, del italiano al español, del español al italiano y, en menor medida, del italiano al francés y del italiano y el español al alemán. En Europa fue leído, principalmente, en italiano ${ }^{5}$, gracias sobre todo a las múltiples ediciones, de la primera (Parma, y también inmediatamente traducida al español) a la última (Nápoles), de su obra mayor, la Historia universal de las Letras y las Ciencias (Origen, progresos y estado actual de toda la literatura).

III

A partir del antecitado estudio sobre la constitución de la Escuela Universalista Española del siglo XVIII, publicado a propósito de la gran exposición bibliográfica celebrada en la Biblioteca Histórica de la Universidad Complutense durante el primer

\footnotetext{
4 Para los problemas de la historiografía ilustrada es imprescindible Ernst Cassirer, quien mejor ha explicado tanto su entidad epistemológica como su circunstancia histórica injustificadamente atacada por el Romanticismo. Pero además, el estudio de Cassirer permite comprobar sobre sí el éxito de la falacia romántica por cuanto él, víctima de la misma, no tiene referencia de la obra de Andrés.

5 Al parecer, la manipulada edición francesa (Histoire Générale des Sciences et de la Littérature, Imprimerie Imperial, París, 1805) del volumen inicial y programático de la obra mayor de Andrés apenas tuvo distribución, según ha estudiado el prof. Javier Pérez Bazo.
} 
semestre de $2017^{6}$, ofrecemos aquí los elementos fundamentales que permiten determinar los diferentes sectores de la Escuela Universalista: subescuelas, círculos, esferas, individualidades, comunidades. Las investigaciones a fecha de hoy han permitido ya sin duda el trazado de toda la base de la Escuela, pero es preciso insistir en que aún no lo damos por cerrado, pues la emergencia de una extensa gama compuesta por varias decenas de autores, más sus precedentes inmediatos (y también precursores alejados y, por último, autores relacionados y consecuentes), no ha sido estudiado sino preliminarmente más allá de ciertos núcleos. Esto atañe sobre todo a autores americanos e italianos. Mucho queda por matizar y concretar, sobre todo en lo que se refiere a autores no de primer orden, o a la reinterpretación de algunos importantes y en especial sus discípulos. Muy diferente asunto es cómo se haya podido llegar al siglo XXI en esta situación de los estudios dieciochistas, pero eso es lo que hay, por muy grave que pueda parecer. No es ahora ocasión de entrar en esta problemática heredada en torno a la Ilustración.

Desde 1987 se ha calificado con el término "universalista" a estos autores o sus obras he insistido sobre Juan Andrés, Lorenzo Hervás y Antonio Eximeno como las grandes figuras de la Escuela (Aullón, Géneros 48). Ahora bien, a éstos se han de sumar muchos otros, centralmente los botánicos y naturalistas, españoles, americanos, filipinos: Cavanilles, Mutis, Clavijero, Molina, Camaño, Concepción, etc. La subescuela mexicana, encabezada por Clavijero, es sin duda la más nutrida y todavía menos examinada. La diferencia consiste en que aquellos tres llevaron a término proyectos metodológicos clave para la construcción cenital del universalismo desde criterios comparatistas, para la ciencia, la cultura, la literatura y la Historia moderna de las ideas. Pero por ejemplo, Clavijero es quien aporta, directamente, la gran visión de la población indígena, el otro lado humano que permite cerrar el horizonte universalista respecto de ese término fundamental.

La solidaria y convergente formación del núcleo de la Escuela y su entorno, la evidencia universalista de su concepción intelectual, al amparo del comparatismo humanístico de raigambre clásica, asociado al proyecto de una ciencia futura anclada en la filosofía empírica y la física newtoniana según ya establecía Andrés colegiadamente en el que denominamos "primer programa epistemológico" de la Escuela Universalista

\footnotetext{
${ }^{6}$ Exposición enero-junio de 2017, Biblioteca Histórica "Marqués de Valdecilla", de la Universidad Complutense:http://www.aecid.es/ES/Paginas/Minisite\%20Biblioteca/Difusi\%C3\%B3n/Destacados/0000 99-expo_univers.aspxWeb. 22 febrero 2018.
} 
(Aullón, Escuela 45-62)7 , permite delimitar en realidad un amplio perímetro de acción y confluencias y un amplio número de obras o autores. Esta dimensión extensa, que naturalmente enriquece en sumo grado el valor de las discriminaciones acerca de los "escuelistas", al tiempo que les otorga una relevancia expansiva de insospechado alcance, requiere por ello mismo un examen razonado y autolimitativo.

De atender a lo más primario, el hecho de que los universalistas en su mayoría fuesen jesuitas describe una circunstancia importante, pero no más que una circunstancia y parcial, siendo además el caso que el haber focalizado con preeminencia tal cosa relativa a orden religiosa ha contribuido gravemente a cegar otras posibilidades de estudio de base propiamente epistemológica, sobre todo las relativas a la especificidad de las portentosas investigaciones por nuestros escuelistas realizadas e incluso la misma determinación del concepto 'universalista', cuyo anclaje es culturalmente general pero fueron nuestros autores quienes decidieron culminar a su modo. Por lo demás, es de advertir acerca de que la circunstancia de la profesión religiosa predeterminó la condición misionera de algunos de los autores, su diseminación por las Indias, su altísima movilidad geográfica desde América a Asia y cómo la operación política de la expulsión española de los jesuitas en 1767, que había de conducir a Italia como centro, recreó una especial modalidad de transterramiento cuya fenomenografía sociohistórica y circunstanciación en individuos concretos, en su realidad vital, tuvo como resultado factores reconocidamente adecuados para la expansión intercultural y el incremento de la perspectiva comparatista. Si ese campo y esos estudios, además de un argumento teórico humanístico, venían precedidos por la gran comunidad política y cultural hispánica, no sólo religiosa, sea de notar que importantes universalistas como Franco Dávila o Javier de Cuéllar no fueron jesuitas, ni misioneros ni sacerdotes. Se ha de advertir parejamente acerca de la actividad expedicionaria, política y concerniente a los grandes viajes comerciales y científicos cuya relevancia es manifiesta y a día de hoy suficientemente conocida. Pero permítaseme cerrar el bucle del argumento: una cosa es el posible tratamiento historiográfico aplicable a la historia de las expediciones y otra bien distinta la historia de las ideas, aunque el impulso expedicionario pueda ser también objeto de esta última.

\footnotetext{
${ }^{7}$ Para el estudio de ese primer programa, Prospectus Philosophiae Universae (Ferrara, Iosepho Rinaldi, 1773), véase el ya citado La Escuela Universalista Española del siglo XVIII (45-62). También para lo que sigue.
} 
En su conjunto, la Escuela Universalista describe confluencias científicas especificables, por una parte, mediante una serie de esferas o "círculos" de relación de los autores concernidos, a veces en una suerte de "comunidad", a su vez en intersección con los campos disciplinares o temáticos (bibliográfico, lingüístico, botánico, físico, americanista, filipinista...), de lo cual en ocasiones se sigue la posible especificación de subescuelas y subgrupos. A su vez existen, como no podía ser de otro modo, múltiples relaciones, ya como antecedentes próximos, es decir "autores relacionados", también subsecuentes, o bien como antecedentes más o menos lejanos, es decir "precursores". Salvo alguna excepción, apenas entraremos aquí en las repercusiones y consecuentes. En cualquier caso y según nuestro argumento, la Escuela Universalista es precursora insospechada, como gran antecedente intelectual, esto es en su mejor posible sentido, de la Globalización. Todo ello especifica muy diferentes formas de la continuidad y las formas del saber.

Andrés y Hervás configuraron no ya obras de totalización enciclopédica culminante sino dos auténticas comunidades de estudiosos, dos comunidades intelectuales y científicas internacionales. Con preferencia me referiré ahora a los círculos de relación de la treintena aproximada de autores que cabe razonadamente determinar como "escuelistas", sus caracteres más generales, tendencias y grupos y tematizaciones; también a los autores con más eficiencia "relacionados", incluyendo los precedentes y algunos subsecuentes. Entenderemos por "relacionados" aquellos autores discriminables mediante algún relevante concepto de universalismo o de vinculación a éste, ya por distinción analógica o ya por fehaciente integración participativa. Esta categoría de "relacionados" incorporará un entorno de hasta aproximadamente un siglo a la redonda, si se me permite la expresión. Se indicarán pues las principales evoluciones $\mathrm{y}$, por último y sin discontinuidad, la discriminación incluso de los antecedentes lejanos. Según lo referido, y siguiendo naturalmente el método comparatista, se entenderá que las relaciones pueden establecerse como 'de facto' o bien 'por analogía'. El resultado habrá de ser el establecimiento de una nómina muy matizada de autores de la Escuela, así como de aquellos otros relacionados o relacionables, y ciertos antecedentes o precursores notables (Aullón, Escuela).

Se podrá comprobar que se trata de una gama de individuos con frecuencia calificable, al modo de la comparatística, de talentos dobles o, por mejor decir, múltiples, cosa que otorga extraordinaria solidez y amplitud generalista a la Escuela y permite por otra parte considerar el aspecto de pluralidad tematológica como 
caracterizador de la misma. También se trata de sacerdotes y profesores, preceptores, misioneros, empresarios y expedicionarios, funcionarios de la administración, y frecuentísimamente viajeros, a menudo forzadamente abocados al viaje por destierro, pero en cualquier caso cosmopolitas de concepto y de hecho constructores de una imagen más plena y totalizadora del mundo humano y científico y del Universo.

Será conveniente considerar también el fenómeno revelador de que la América española y las Filipinas en Asia configuraron no un "laboratorio", según es grato decir metodológicamente a posteriori a cierta sociología, sino una auténtica escuela de aprendizaje y proyección universalista que determinó un enriquecimiento prodigioso de la cultura hispánica y moderna. Es exigible, a vista de las obras, que esto sea debidamente reconocido.El método general aplicado para establecer la adscripción de los miembros y, a fin de cuentas, las dimensiones de la Escuela ha consistido en determinar, de manera separada o combinada, tres aspectos sobre los autores: a) entidades de concepto y temáticas o disciplinares; b) relaciones intelectuales acerca de obras e ideas, o entre individuos pero en tanto conducentes a obras e ideas; c) todo ello sobre la base de un pensamiento o ideación de universalismo humanístico, científico, literario e histórica y disciplinarmente integrador $\mathrm{y}$, por principio, radicalmente internacionalista o intercontinentalista. Si el criterio de integración es en todo sentido de evidente ambición tradicional humanística o moderna globalista, tanto para la ciencia como para la historia, el criterio de internacionalismo e intercontinentalismo, también notablemente revelador, atiende sin embargo a un aspecto que no sólo es remisible a diversos planos de la realidad sino que en ocasiones requiere ser concretamente matizado. El hecho es que la aplicación a un objeto discernible como universalista o globalista, internacionalista o intercontinentalista no cabe ser definida desde las limitaciones simplemente establecidas desde una posición cultural o geográfica de localización meramente opuesta por distante, sino mediante determinación de la propia naturaleza y función del objeto que se toma a consideración. Por lo demás, cada decisión concreta adoptada acerca de la inclusión de autores, viene precedida por un riguroso y puntual análisis que aquí no será necesario explicitar más allá de rasgos de concepto esenciales o bibliográficamente muy caracterizadores.

Es de observar el eficiente dominio de la disposición de relaciones intelectuales a modo de red o en comunidad. Esto sin duda propiciado a su vez por el predominio de individuos religiosos y, entre éstos, de jesuitas. Pero se ha de insistir en el permanente criterio de observar obras y el resultante de ideas por encima de circunstancias, pues 
precisamente lo que nos guía es la historia y evaluación de aquéllas y no de éstas. A través de las Cartas familiares de Andrés se hace posible iluminar la imagen de un fresco de individualidades intelectuales e incluso ciertas agrupaciones de éstas, bien que a menudo espigadas de entre el mundo y la clase intelectual italiana. Según decía Franco Venturi, teniendo presente la censura que se solía aplicar a la Compañía de Jesús, en la mencionada obra de Andrés más se deja ver la actividad de una comunidad de doctos alerta y atenta a todo lo circundante que no una organización más o menos secreta (Venturi 266).

Es conveniente discernir, por encima de "individuo" o "individualidad", entre los aspectos de "comunidad intelectual" y "tendencia intelectual", si bien ambos cabe sean tomados, según sus diferentes posibilidades, en sentido puntual o bien dilatado en el tiempo. La fuerza de la "tendencia" de manera concreta será discriminable sobre todo y paradigmáticamente a partir de las grandes cabezas "individuales" de las formaciones de comunidad, es decir en sus obras o en el estro de ellas, al igual que en las grandes figuras individuales característica o dominantemente autónomas, así como por otra parte en los lineamientos cuya corriente precede o subsigue en el tiempo y hace especialmente necesario discriminar obras y autores "relacionados" de uno u otro modo e incluso, más allá del mundo estrictamente coetáneo, "precursores".

La consideración de relaciones o autores relacionados distantes en el tiempo, particularmente en el caso de la obra de Andrés, no sólo sugiere antecedentes o precursores de mucho relieve sino que vendría a constituir un verdadero entramado histórico-teórico. A estos efectos me limitaré a referir muy selectivamente ciertos elementos, ya se trate estrictamente de elementos precursores o bien, en algunos casos, de tomas de partido o similar. Es preciso advertir cómo, en Italia, la obra de Andrés estatuye un extenso y penetrante fenómeno editorial e intelectual cuando menos hasta mediados del siglo XIX. El hecho es que fue usada como tratado manual en conjunto y por partes incluso en sucesivas ediciones parciales y por compendio. También fue objeto de plagios varios de distinta índole, y de reproducciones parciales, más o menos honestas, junto a otros textos. De todo ello disponemos de datos y ejemplos, si bien aún a falta de completo estudio.

En lo que se refiere a la cultura antigua greco-latina, materia sobre la cual la valoración de Andrés es superior a la de la asiática, tan de moda en aquel tiempo, la operación de nuestros autores consistió característicamente en un hábil y decidido maridaje de humanismo antiguo y ciencia moderna. Esta continuidad de transmisión es 
en realidad conseguida mediante el desenvolvimiento del método comparatista y el valor sostenido de los clásicos. Si esto se apoya sobremanera en el padre de la Crítica literaria, Dionisio de Halicarnaso, así como en el desarrollo del parangón greco-latino y de diferente lado en Cicerón y demás, el sentido enciclopédico y de totalización del conocimiento, según puede comprobarse notablemente en Andrés, Hervás y otros, tiene en Plinio el Viejo la principal y reconocida antecedencia o base precursora, que a su vez perpetuaría la tradición española hasta Menéndez Pelayo, y posteriormente interrumpida al igual que la constituida por la propia Escuela Universalista. Aparte casos como el de Casiodoro, o desde luego Petrarca, uno de los ejemplos humanísticos poligráficos por antonomasia, y Escalígero, en términos científicos la obra y figura clave es la de Galileo, seguida de Newton y sus precedentes, muy intensamente en Andrés desde tiempos de Gandía y Ferrara. En cuanto a la tradición española, además de Juan Luis Vives, paradigma antiescolástico irrenunciable, los precursores españoles son sobre todo Alfonso X el Sabio, así como en general el internacionalismo de la denominada Escuela de Traductores de Toledo y su entorno, y san Isidoro de Sevilla. Desde el punto de vista globalista e intercontinentalista es de señalar en el primer cuarto del siglo XVI la figura del tripulante italiano Antonio de Pigafetta, cuyo diario es el mejor relato directo del viaje de circunnavegación del Planeta por Fernando de Magallanes y Juan Sebastián Elcano. Hay que subrayar dentro del mismo siglo la poco conocida Escuela de Traductores de Manila y su cabeza el misionero dominico, cosmógrafo y filólogo Juan Cobo, primer sinólogo europeo (Beng Sim Po Cam o Espejo Rico del Claro Corazón, 1592) ${ }^{8}$, mediante el cual enlaza magnífica y universalistamente la tradición escriturística y traductológica que va de San Jerónimo a Erasmo gracias a la unión de filología profana y sacra que culmina Andrés ${ }^{9}$. Las diferentes perspectivas en el estudio de las lenguas acrisolado en las obras de Hervás, Andrés y Eximeno constituyen la renovación de la tradición grecolatina y de la Gramática en su más alto sentido de

\footnotetext{
${ }^{8}$ Hay ediciones modernas: ed. de Carlos Sanz, Madrid, Librería General Victoriano Suárez, 1959. Es edición especial conmemorativa, facs. bilingüe y traducción. Existe edición anterior de 1924, aquí seguida, y posterior también bilingüe de Li-mei Liu (2005) y de Zhao Zhenjiang (2012).

${ }^{9}$ Una interpretación de esta visión de la tradición humanística puede verse en el segundo capítulo de P. Aullón de Haro, "La ideación del humanismo y la problematización humanística de nuestro tiempo", que abre Teoría del Humanismo (25-101). La obra mayor de Andrés mantiene una estricta observancia acerca de los textos necesarios a su investigación, es decir, aparte de la lengua origen de la obra, la posible existencia de su traducción y estudio. La pérdida de esta observancia ha sido uno de los factores determinantes de la progresiva decadencia de la historiografía literaria contemporánea.
} 
Filología general, tal establece Andrés, quien vincula de hecho y de manera notable Filología y Anticuaria ${ }^{10}$.

La relación entre los universalistas y la otra gran Escuela española, la de Salamanca del Siglo de Oro, se establece sobre todo a partir del internacionalismo político y jurídico inaugurado por Francisco de Vitoria. El humanismo cristiano universalista profesado por él, manifiesto en el respeto hacia las poblaciones indígenas, el mundo de las colonias y su evangelización, se traduce en nuestros autores en un proyecto más específicamente historiográfico, cartográfico y lingüístico, sobre todo en los casos de Hervás, Joaquín Camaño (La Rioja, Argentina 1737 - Valencia, 1820) (vid. Piciulo) y Juan Ignacio Molina (Villa Alegre, Chile 1740 - Bolonia 1829). La obra americanista de Molina, bien conocida, atañe especialmente a Chile y se desarrolló principalmente en el último cuarto del siglo XVIII, es decir, en concomitancia con la eclosión de la conocida "Disputa sobre América", de la que fueron protagonistas diferentes intelectuales como Buffon, De Pauw, Raynal o Robertson (vid. Gerbi).

Decisiva es la influencia, sobre todo en el caso de Andrés y Eximeno, de los Novatores valencianos (en concreto Tomás Vicente Tosca, 1651-1723, y Juan Bautista Corachán, 1661-1741) y del profesorado de la Universidad de Cervera, compuesto prevalentemente por intelectuales jesuitas, entre los que mencionamos por importancia Mateo Aymerich (Bordils, Gerona, 1715 - Ferrara, 1799) y el jurista José Finestres (Barcelona, 1688 - Cervera, 1777).

Un aspecto decisivo de la Escuela Universalista es sin duda el ámbito bibliográfico y biblioteconómico. Miguel de Casiri de Gartia (Tripoli, 1710 - Madrid, 1791), arabista discípulo de Francisco Rábago, es autor de un importantísimo catálogo de las obras árabes conservadas en la Biblioteca del Escorial. La tesis andresiana sobre el papel de la cultura árabe en el progreso de la literatura y las ciencias en el Medievo europeo debe mucho a las noticias y datos ofrecidos por Casiri. En el campo de la bibliografía, al que Andrés y Hervás dedicaron muchos trabajos, se inscriben también las obras de Esteban Terreros y Pando (Trucios, Vizcaya, 1707 - Forlì, 1782) y Ramón (o Raimundo) Diosdado Caballero (Mallorca, 1740 - Roma, 1829).

En cuanto a la Historia natural, Jorge Juan (Monforte, Alicante, 1713 - Madrid, 1773) y Antonio Ulloa (Sevilla, 1716 - Cádiz, 1975), gracias a sus investigaciones astronómicas, geográficas y geodésicasdesarrolladas en América del Sur, son los

\footnotetext{
${ }^{10}$ Véase el Estudio incluido en Juan Andrés, Furia. Disertación sobre una inscripción romana (13-93).
} 
precursores inmediatos de la Escuela, la cual cuenta con varios naturalistas y botánicos de relieve, así Franco Dávila (Guayaquil, 1711 - Madrid, 1786), impulsor del Real Gabinete de Historia Natural di Madrid (1776), Antonio José Cavanilles (Valencia, 1745 - 1804), quien mantuvo un denso intercambio epistolar con Andrés, o José Celestino Mutis. El referido jesuita y americanista chileno Juan Ignacio Molina, precursor del evolucionismo y bien reconocido por Andrés,es autor de un Saggio sulla storia naturale del Chili (1782).

En lo relativo a la esfera de influencias de Hervás, son muchos los colaboradores que contribuyeron a su Catálogo de las lenguas. Recordamos a Juan Clímaco Salazar (Caravaca, Murcia 1744 - Hellín, Albacete, 1815), Francisco Javier Clavijero (Veracruz, México, 1731 - Bolonia, 1787), Pedro José Márquez (Guanajuato, 1741 Ciudad de México, 1820), José Lino Fábrega (Tegucigalpa, 1746 - Vitorchiano, 1797), Juan Nuix (Torà, Lérida, 1740 - Ferrara 1783) o Joaquín Camaño (La Rioja, Virreinato de La Plata, 1737 - Valencia, 1821). Entre los colaboradores filipinos, destacan Bernardo Bruno de la Fuente (Villanueva de la Jara, Cuenca, 1732 - Faenza, 1807) y Juan Antonio de Tornos (Sigüenza, 1727 - Roma, 1802). Clavijero será quien más que otros llevará a cabo varias investigaciones históricas clave para el conocimiento de la realidad americana, como la Historia antigua de México o la Historia de California.

Más cercanos a Andrés fueron, en cambio, su hermano Carlos (Planes, Alicante, 1753 - Valencia, 1820), traductor y difusor de sus obras en España, Antonio Pinazo (Alpuente, Castellón, 1750 - Mantua, 1820), poeta interesado por la divulgación artística de la Astronomía y la Física, el matemático Antonio Ludeña (Almusafes, Valencia, 1740 - Cremona, 1820), Joaquín Millás (Zaragoza, 1746 - 1809), teórico de la estética, metafísico y misionero en Paraguay, y Juan Bautista Colomés (Valencia, 1740 - Bolonia, 1808). La curiosa figura intelectual del erudito Carl Grosse, alias Eduardo Romeo conde de Vargas-Bedemar (Magdeburgo 1768 - Copenhague, 1847), es ejemplo del cosmopolitismo y la poligrafía típica de buena parte de los universalistas: además de textos literarios, escribió artículos y monografías sobre temáticas inherentes a la estética, la geología, la física o la matemática.

En el ámbito de la musicología y el estudio de las artes, y por tanto más relacionados con Antonio Eximeno, Vicente Requeno (Calatorao, Zaragoza, 1743 Tivoli 1811) escribió ensayos sobre pintura y música de la Antigüedad, mientras que Josef Pintado (Sevilla, 1741 - 1819) y Buenaventura Prats (Tarragona, 1749 - Manresa, Barcelona, 1825) se ocuparon de temas más propiamente musicológicos. Juan Bautista 
Muñoz $^{11}$ (Museros, Valencia, 1745 - Madrid, 1799), americanista y fundador del Archivo General de Indias, fuediscípulo directo de Eximeno, el gran teórico universalista de la música.

\section{Bibliografía}

Andrés, Juan. Cartas familiares (Viaje de Italia). Eds. I. Arbillaga y C. Valcárcel, P. Aullón de Haro. II. Madrid: Verbum, 2004.

Andrés, Juan. Epistolario. Ed. L. Brunori. III. Valencia: Biblioteca Valenciana, 2006.

Andrés, Juan. Estudios Humanísticos. Eds. P. Aullón de Haro, E. Crespo, J. García Gabaldón, D. Mombelli y F. J. Bran. Madrid: Verbum, 2017.

Andrés, Juan. Furia. Disertación sobre una inscripción romana. Eds. P. Aullón de Haro y D. Mombelli. Madrid: Instituto Juan Andrés, 2017.

Andrés, Juan. Origen, progresos y estado actual de toda la literatura. Eds. J. García Gabaldón, S. Navarro y C. Valcárcel, P. Aullón de Haro. VI. Madrid: Verbum, 1997-2002.

Aullón de Haro, Pedro. Los géneros didácticos y ensayísticos en el siglo XVIII. Madrid: Taurus, 1987.

Aullón de Haro, Pedro. Teoría del Humanismo. I. Madrid: Verbum, 2010.

Aullón de Haro, Pedro, Ed. Metodologías comparatistas y Literatura comparada. Madrid: Dykinson, 2012.

Aullón de Haro, Pedro. La escuela universalista Española. Madrid: Sequitur, 2016.

Aullón de Haro, Pedro. "Constitución de la Escuela Universalista Española del siglo XVIII". Juan Andrés y La escuela Universalista española. Eds. P. Aullón de Haro y J. García Gabaldón. Madrid: Ediciones Complutense, 2017.

Cassirer, Ersnt. La filosofía de la ilustración. $3^{\mathrm{a}}$ ed. México: FCE, 1972.

Eximeno, Antonio. Del origen y reglas de la música. Ed. A. Hernández Mateos. Madrid: Verbum, 2016.

Gerbi, Antonello. La disputa del Nuevo Mundo. Historia de una polémica 1750-1900. $2^{a}$ ed. México: FCE, 1993.

Muñoz, Juan Bautista. Historia del Nuevo Mundo. Ed. J. Alcina Franch. México: Aguilar, 1975.

Piciulo, Viviana. I gesuiti americani espulsi e Joaquín Camaño (1737-1723). Bolonia: Università di Bologna, 2014.

Venturi, Franco. Settecento riformatore. (I), IV.Turín: Einaudi, 1984.

\footnotetext{
${ }^{11}$ Véase la edición moderna de la Historia del nuevo mundo, por J. Alcina Franch.
} 